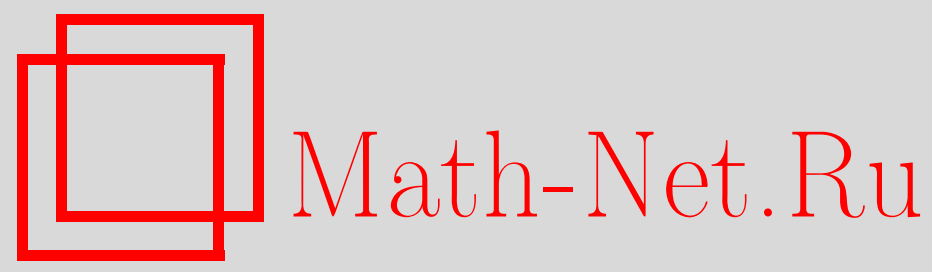

М. М. Глухов, П. Н. Закревский, О коэффициентах аддитивности и аффинности дискретных функций, Дискрет. матем., 2012, том 24, выпуск 1, 30-47

DOI: https://doi.org/10.4213/dm1170

Использование Общероссийского математического портала Math-Net.Ru подразумевает, что вы прочитали и согласны с пользовательским соглашением http://www . mathnet.ru/rus/agreement

Параметры загрузки:

IP: 54.197 .130 .99

26 апреля 2023 г., 15:28:09 


\title{
О коэффициентах аддитивности и аффинности дискретных функций
}

\author{
() 2012 г. М. М. Глухов, П. Н. Закревский
}

\begin{abstract}
Вводятся и изучаются коэффициенты аддитивности и аффинности функций на конечных группах. При этом особое внимание уделяется булевым функциям и бентфункциям.
\end{abstract}

\section{1. Введение}

Будем использовать следующие обозначения:

$B^{A}$ есть множество всех функций, определенных на $A$ со значениями в $B$;

$\|f\|=|\{a \in A: f(a) \neq 0\}|$ есть вес функции $f \in B^{A}$ при условии, что $A=(A ;+)$ есть группоид с нулем;

$N_{b}^{f}=\{a \in A: f(a)=b\}$ для $f \in B^{A}, a \in A, b \in B ;$

$V_{n}$ есть линейное пространство векторов-строк длины $n$ над полем $P=G F(2)$,

$V_{n}^{*}=V_{n} \backslash\{0\}$

$F_{n}$ есть множество всех булевых функций от $n$ переменных;

$L_{n}$ есть множество всех линейных булевых функций от $n$ переменных;

$A_{n}$ есть множество всех аффинных булевых функций от $n$ переменных;

,,+- 0 есть операции сложения, вычитания и нулевой элемент во всех используемых алгебрах.

В работах по дискретным функциям, связанных с криптографическими приложениями, большое внимание уделяется вопросам приближения функций над конечными полями и кольцами линейными функциями. Развитие этого направления началось, в основном, с исследования булевых функций [8].

Один из основных методов сравнения булевой функции с линейными функциями основан на использовании статистической структуры функции. Напомним (см. [8]), что статистической структурой булевой функции $f\left(x_{1}, \ldots, x_{n}\right)$ называется набор из $2^{n}$ чисел $\Delta_{a}^{f}, a \in V_{n}$, определяемых формулами

$$
\frac{\|f(x)-(a, x)\|}{2^{n}}=\frac{1}{2}-\frac{\Delta_{a}^{f}}{2^{n}},
$$


где $a=\left(a_{1}, \ldots, a_{n}\right) \in V_{n}, x=\left(x_{1}, \ldots, x_{n}\right) \in V_{n}$ и

$$
(a, x)=a_{1} x_{1}+\ldots+a_{n} x_{n} .
$$

Отсюда находим, что

$$
\Delta_{a}^{f}=2^{n-1}-\|f(x)-(a, x)\|
$$

Если ввести расстояние между функциями как расстояние Хэмминга между столбцами табличных заданий этих функций при одном и том же упорядочении векторов-аргументов, то величина $\|f(x)-(a, x)\|$ будет расстоянием Хэмминга между функциями $f(x)$ и $(a, x)$. Значит, элементы $\Delta_{a}^{f}$ статистической структуры функции $f$ будут определять расстояния от функции до линейных функций и тем самым оценивать близость или удаленность функции $f$ от линейных функций.

Еще один подход к сравнению булевых функций из $f \in F_{n}$ с линейными основан на использовании характеров группы $G=\left(V_{n} ;+\right)$ над полем комплексных чисел С. При этом вместо функции $f$ рассматривают так называемую характеристическую для нее функцию $f_{b}(x)=\chi_{b}(f(x))$, где $\chi_{b}-$ комплексный характер аддитивной группы $H=\{0,1\}$ поля $G F(2)$, соответствующий элементу $a$. Так как $\chi_{b}(x)=(-1)^{b x}$, то $f_{b}(x)=(-1)^{b f(x)}$, то есть $f_{0}(x)=1$, а $f_{1}(x)=(-1)^{f(x)}$. Разложим функцию $f_{1}$ в ряд Фурье по характерам $\eta_{a}$ группы $G$ :

$$
f_{1}(x)=\sum_{a \in G} C_{a}^{f} \eta_{a}(x) .
$$

Систему комплексных чисел $C_{a}^{f}$, где $a \in G$, называют спектром функции $f$. Из ортогональности характеров в пространстве всех функций из $\mathbf{C}^{G}$ над полем $\mathbf{C}$ со скалярным произведением

$$
(f, g)=\sum_{x \in G} f(x) \overline{g(x)}
$$

следует, что

$$
C_{a}^{f}=\frac{1}{|G|} \sum_{x \in G} f_{1}(x) \bar{\eta}_{a}^{G}(x) .
$$

Если $x=\left(x_{1}, \ldots, x_{n}\right), a=\left(a_{1}, \ldots, a_{n}\right)$, то $\eta_{a}=(-1)^{(a, x)}$ и

$$
C_{a}^{f}=\frac{1}{|G|} \sum_{x \in G}(-1)^{f(x)-(a, x)} .
$$

Заметим еще, что для функции $f \in F_{n}$ имеет место равенство

$$
\|f(x)-(a, x)\|=2^{n-1}-\frac{1}{2} \sum_{x \in V_{n}}(-1)^{f(x)-(a, x)} .
$$

Отсюда и из (1) получаем соотношение

$$
\Delta_{a}^{f}=2^{n-1} C_{a}^{f}
$$

Таким образом, близость булевой функции к линейным функциям можно характеризовать и элементами статистической структуры, и элементами спектра. 
Булева функция $f \in F_{n}$ называется минимальной, а также бент-функцией, если $\left|\Delta_{a}^{f}\right|=2^{n / 2-1}$ для любого $a \in V_{n}$. Следовательно, для бент-функции $f$ от $n$ переменных выполняется условие

$$
\|f(x)+(a, x)\|=2^{n-1} \pm 2^{n / 2-1} .
$$

В дальнейшем многими авторами понятия статистической структуры, спектра, бентфункции и другие понятия для булевых функций обобщались естественным образом на функции, определенные на различных конечных полях, кольцах, группах (см., например, $[1,3,4])$. В ряде таких работ рассматривался, в частности, способ сравнения функций на группах с линейными функциями и гомоморфизмами, основанный на свойстве

$$
f(a+b)=f(a)+f(b) .
$$

Пусть $(G ;+)$ и $(H ;+)$ есть конечные группы, и $H^{G}$ есть множество всех функций, определенных на $G$ со значениями в $H$. Заметим, что функции над конечным простым полем, обладающие свойством (3) при любых $a, b \in G$, являются линейными. Поэтому гомоморфизмы групп являются аналогами линейных функций над простыми конечными полями. В работе [7] гомоморфизмы групп называются линейными функциями, а свойство (3) положено в основу теста на близость функции $f$ к линейной функции. В последующие годы этому тесту линейности был посвящен ряд работ тех же и других авторов (см. $[5,6,9])$. В них, в частности, исследовалась величина

$$
\operatorname{Err}(f)=\mathbf{P}(f(u)+f(v) \neq f(u+v)),
$$

которую можно трактовать как вероятность отклонения функции $f$ от гомоморфизма при равновероятном и независимом выборе элементов $u$ и $v$ из группы $G$. Одна из основных задач, рассматриваемых в указанных работах, заключалась в установлении соотношений между величиной $\operatorname{Err}(f)$ и расстоянием функции $f$ от класса линейных функций (гомоморфизмов).

Заметим, что этот подход сравнения функций с гомоморфизмами для функций от $n$ переменных над кольцами вычетов и конечными полями рассматривался ранее А. С. Амбросимовым (1981 г.). В частности, им в обоих этих случаях для любого целого числа $l>1$ были найдены формулы вероятности аддитивности $\mathbf{P}\left(f\left(\sum_{i=1}^{l} x_{i}\right)=\sum_{i=1}^{l} f\left(x_{i}\right)\right)$ функции $f$ в общем случае, а также в случаях, когда $f$ является бент-функцией. Продолжая это направление исследований, мы введем следующие определения.

Определение 1. Коэффициентом аддитивности функции $f \in H^{G}$ назовем число

$$
A d(f)=\frac{\left|\left\{(x, y) \in G^{2}: f(x+y)=f(x)+f(y)\right\}\right|}{|G|^{2}} .
$$

Определение 2. Коэффициентом аффинности функции $f \in H^{G}$ назовем число

$$
A f(f)=\max _{a \in H} \frac{1}{|G|^{2}}\left|\left\{(x, y) \in G^{2}: f(x+y)=f(x)+f(y)+a\right\}\right| .
$$

В данной статье, используя идеи А. С. Амбросимова, и обозначения из работы [4] В. И. Солодовникова, мы находим выражение коэффициента $A d(f)$ через спектр функции $f$ при условии, что группы $G, H$ являются прямыми суммами нескольких экземпляров одной и той же циклической группы. Частными случаями полученного утверждения являются теоремы Амбросимова о функциях над полями и кольцами вычетов при $l=2$.

Далее в статье основное внимание уделяется нахождению параметров $A d(f)$ и $A f(f)$ для функций из некоторых классов булевых функций. 


\section{2. О коэффициенте аддитивности для дискретных функций на абелевых группах}

Непосредственно из определения коэффициента аффинности функций из $H^{G}$ легко получить следующие простейшие его свойства.

Предложение 1. Для любой функиии $f \in H^{G}$ имеют место следующие свойства коэффиичиента $A f(f)$ :

(1) $1 /|H| \leqslant A f(f) \leqslant 1$;

(2) $A f(f)=1 \Longleftrightarrow f$ есть афффинная функиия;

(3) $A f(f)=A f(f+c)$ для всех $c \in H$;

(4) $A f(f)=A f(f+h)$ для всех $g(x) \in \operatorname{Hom}(G, H)$;

(5) $A f(f)=A f(f \circ \varphi)$ для всех $\varphi \in \operatorname{Aut}(G)$, где о означает композицию функичии.

Для формулировки следующего утверждения введем еще некоторые понятия и обозначения. При изучении функций из $H^{G}$ обычно используются комплексные характеры групп $G$ и $H$. С этой целью по функции $f \in H^{G}$ и элементу $a \in H$ определяют комплекснозначную функцию $f_{a}: G \rightarrow \mathbf{C}$ :

$$
f_{a}(x)=\chi_{a}(f(x)),
$$

где $\chi_{a}$ - комплексный характер группы $H$, соответствующий элементу $a$.

Обозначим через $\eta_{b}^{G}$ характер группы $G$, соответствующий $b$, и разложим функцию $f_{a}$ в ряд Фурье по характерам группы $G$ :

$$
f_{a}(x)=\sum_{b \in G} C_{b}^{f_{a}} \eta_{b}^{G}(x) .
$$

Систему комплексных чисел $C_{b}^{f_{a}}$, где $a \in H, b \in G$, называют спектром функции $f_{a}$. Из ортогональности характеров следует, что

$$
C_{b}^{f_{a}}=\frac{1}{|G|} \sum_{x \in G} f_{a}(x) \bar{\eta}_{b}^{G}(x),
$$

где $\bar{\eta}_{b}^{G}$ - характер, сопряженный с $\eta_{b}^{G}$.

Числа $C_{b}^{f_{a}}$ при любых $a, b \in G$ обладают следующими свойствами:

$$
\begin{aligned}
\left|C_{b}^{f_{a}}\right| & \leqslant 1, \\
\sum_{b \in G}\left|C_{b}^{f_{a}}\right|^{2} & =1, \\
\max \left\{\left|C_{b}^{f_{a}}\right|\right\} & \geqslant \frac{1}{\sqrt{|G|}} .
\end{aligned}
$$

Свойство (4) следует из того, что значениями функций $f_{a}, \chi_{b}^{G}$ являются комплексные корни некоторых степеней из 1 и их модули равны 1. Свойство (5) следует из равенства Парсеваля для унитарного пространства функций $\mathbf{C}^{G}$ со скалярным произведением

$$
S(f, g)=\frac{1}{|G|} \sum_{x \in G} f(x) \bar{g}(x),
$$


поскольку множество характеров $\eta_{b}^{G}, b \in G$, является его ортонормированным базисом. Свойство (6) следует из (5).

Функцию $f \in H^{G}$ называют бент-функцией, если числа $\left|C_{b}^{f_{a}}\right|$ равны между собой при любых $b \in G, a \in H \backslash\{0\}$. Из равенства (6) следует, что для любой бент-функции $f \in H^{G}$ выполняются равенства

$$
\left|C_{b}^{f_{a}}\right|=|G|^{-1 / 2}
$$

Теорема 1. Пусть $f: G \rightarrow H$, где $G=(\mathbf{Z} / q)^{n}, H=(\mathbf{Z} / q)^{t}$ есть прямые суммы аддитивной группь $\mathbf{Z} / q$. Тогда имеет место равенство

$$
A d(f)=\frac{1}{|H|} \sum_{a \in H} \sum_{b \in G} C_{b}^{f_{a}}\left|C_{b}^{f_{a}}\right|^{2} .
$$

Доказательство. Обозначим правую часть равенства (7) через $X$. Так как группа $\mathbf{Z} / q$ циклическая порядка $q$, то ее характер $\varphi_{u}$, соответствующий элементу $u$, определяется равенствами

$$
\varphi_{u}(x)=e_{1}^{u x}
$$

где $e_{1}$ есть фиксированный первообразный корень степени $q$ из 1 . Не теряя общности, можно считать, что для всех прямых слагаемых групп $G, H$ используется один и тот же корень $e^{2 \pi i / q}$. Тогда из (2) и (3) получаем, что

$$
\begin{aligned}
C_{b}^{f_{a}} & =\frac{1}{|G|} \sum_{x \in G} f_{a}(x) \bar{\eta}_{b}^{G}(x)=\frac{1}{|G|} \sum_{x \in G} \chi_{a}(f(x)) \bar{\eta}_{b}^{G}(x) \\
& =\frac{1}{|G|} \sum_{x \in G} e^{(2 \pi i / q)((a, f(x))-(b, x))},
\end{aligned}
$$

где для элементов $u=\left(u_{1}, \ldots, u_{r}\right) \in(\mathbf{Z} / q)^{r}, v=\left(v_{1}, \ldots, v_{r}\right) \in(\mathbf{Z} / q)^{r}$ под $(u, v)$ понимается число $u_{1} v_{1}+\ldots+u_{r} v_{r} \bmod q$. Учитывая, что элемент, сопряженный с корнем из 1 , совпадает с обратным к нему, получим, что

$$
\begin{aligned}
X & =\frac{1}{|H||G|^{3}} \sum_{a \in H} \sum_{b \in G} e^{(2 \pi i / q)((a, f(x)+f(y)-f(z))-(b, x+y-z)))} \\
& =\frac{1}{|H||G|^{3}} \sum_{a \in H} \sum_{x, y, z \in G} e^{(2 \pi i / q)((a, f(x)+f(y)-f(z))} \sum_{b \in G} e^{-(2 \pi i / q)(b, x+y-z)} .
\end{aligned}
$$

Легко проверить, что для любого $u_{1} \in\{1, \ldots, q-1\}$ выполняется равенство

$$
\exp \left\{\frac{2 \pi i}{q} u_{1} 0\right\}+\exp \left\{\frac{2 \pi i}{q} u_{1} 1\right\}+\ldots+\exp \left\{\frac{2 \pi i}{q} u_{1}(q-1)\right\}=0 .
$$

Следовательно, последняя сумма в (8) равна нулю при $x+y \neq z$, и равна $|G|$ при $x+y=z$. Учитывая этот факт и поменяв в (8) порядок суммирования, получим, что

$$
X=\frac{1}{|H||G|^{2}} \sum_{x, y \in G} \sum_{a \in H} e^{(2 \pi / i) q(a, f(x)+f(y)-f(x+y))} .
$$


По тем же соображениям, что и выше, последняя сумма равна 0 , если $f(x)+f(y) \neq$ $f(x+y)$, и равна $|H|$ в противном случае. Значит,

$$
X=\frac{1}{|G|^{2}} \sum_{\substack{x, y \in G \\ f(x)+f(y)=f(x+y)}} 1=\operatorname{Ad}(f),
$$

и теорема доказана.

Заметим, что формула (7) является весьма громоздкой: в ней участвует весь спектр рассматриваемой функции. Однако в качестве следствий из нее можно получить достаточно простые формулы для бент-функций.

Следствие 1. Если функциия $f$ из теоремы 1 является бент-функцией, то

$$
A d(f)= \begin{cases}\frac{1}{|H|}+\frac{|H|-1}{|G||H|}, & f(0)=0, \\ \frac{1}{|H|}-\frac{1}{|G||H|}, & f(0) \neq 0 .\end{cases}
$$

Доказательство. Введем обозначения $H \backslash\{0\}=H^{*}$. Согласно равенству (7)

$$
\begin{aligned}
A d(f) & =\frac{1}{|H|} \sum_{a \in H} \sum_{b \in G} C_{b}^{f_{a}}\left|C_{b}^{f_{a}}\right|^{2} \\
& =\left.\frac{1}{|H|} \sum_{a \in H^{*}} \sum_{b \in G} C_{b}^{f_{a}}\left|G^{-1}+\frac{1}{|H|} \sum_{b \in G} C_{b}^{f_{0}}\right| C_{b}^{f_{a}}\right|^{2} .
\end{aligned}
$$

Из определения функции $f_{a}$ видно, что $C_{b}^{f_{0}}=0$, если $b \neq 0$ и $C_{b}^{f_{0}}=1$, если $b=0$. Отсюда следует, что

$$
\sum_{b \in G} C_{b}^{f_{0}}\left|C_{b}^{f_{a}}\right|^{2}=1,
$$

и поэтому

$$
\sum_{a \in H^{*}} \sum_{b \in G} C_{b}^{f_{a}}=\sum_{a \in H} \sum_{b \in G} C_{b}^{f_{a}}-1 .
$$

Далее,

$$
\begin{aligned}
\sum_{a \in H} \sum_{b \in G} C_{b}^{f_{a}} & =\sum_{a \in H} \sum_{b \in G} \sum_{x \in G} \exp \left\{\frac{2 \pi i}{q}((a, f(x))-(b, x))\right\} \\
& =\sum_{x \in G} \sum_{a \in H} \exp \left\{\frac{2 \pi i}{q}(a, f(x) t)\right\} \sum_{b \in G} \exp \left\{-\frac{2 \pi i}{q}(b, x)\right\} \\
& =|G| \sum_{a \in H} \exp \left\{\frac{2 \pi i}{q}(a, f(0))\right\} .
\end{aligned}
$$

Остается заметить, что

$$
\begin{array}{ll}
\sum_{a \in H} \exp \left\{\frac{2 \pi i}{q}(a, f(0))\right\}=0, & f(0) \neq 0, \\
\sum_{a \in H} \exp \left\{\frac{2 \pi i}{q}(a, f(0))\right\}=|H|, & f(0)=0 .
\end{array}
$$

Подставив найденные величины в (10), получим доказываемое утверждение. 
Замечание 1. Для случая $G=(\mathbf{Z} / q)^{n}, H=\mathbf{Z} / q$. формулы (7) и (8) совпадают с соответствующими формулами А. С. Амбросимова при $l=2$ для функций от $n$ переменных над $\mathbf{Z} / q$, а при $G=(\mathbf{Z} / p)^{n t}, H=(\mathbf{Z} / p)^{t}-$ с его формулами для функций от $n$ переменных над полем $G F\left(p^{t}\right)$.

\section{3. О коэффициентах аддитивности и аффинности для булевых функций}

Так как в данном пункте будут рассматриваться только булевы функции, как правило, будем называть их просто функциями.

При изучении булевых функций из $F_{n}$ в качестве $G$ используется группа $\left(V_{n} ;+\right)$, которая отождествляется с пространством $V_{n}$, а в качестве $H$ используется аддитивная группа поля $P=G F(2)$.

Из утверждения 1 и следствия теоремы 1 получаем следующие утверждения для булевых функций.

Следствие 2. Для любой функции $f \in F_{n}$ имеют место следующие свойства коэффициента $A f(f)$ :

(1) $1 / 2 \leqslant A f(f) \leqslant 1$;

(2) $A f(f)=1 \Longleftrightarrow f \in L_{n}$ или $f+1 \in L_{n}$;

(3) $A f(f)=A f(f+1)$;

(4) $A f(f)=A f(f+g)$ для всех $g(x) \in L_{n}$;

(5) $A f(f(x))=A f(f(\varphi(x)))$ для любого линейного преобразования $\varphi$ пространства $V_{n}$.

Следствие 3. Для любой функциии $f \in F_{n}$

$$
A d(f)=\frac{1}{2}+\frac{1}{2^{3 n-2}} \sum_{a \in V_{n}}\left(\Delta_{a}^{f}\right)^{3} .
$$

Следствие 4. Если $f-$ бент-функция из $F_{n}$, то

$$
A f(f)=\frac{1}{2}+\frac{1}{2^{n+1}} .
$$

Для нахождения параметра $A d(f)$, исходя из его определения, необходима проверка условия $f(a+b)=f(a)+f(b)$ для всех пар $(a, b) \in V_{n}^{2}$. Следующее утверждение позволяет уменьшить полный перебор, а также может оказаться полезным для решения некоторых других вопросов, связанных с коэффициентами аффинности и аддитивности функций.

Теорема 2. Пусть $f \in F_{n},\|f\|=k, N_{1}^{f}=\left\{c_{1}, \ldots, c_{k}\right\}, M-$ множество из $m$ неупорядоченных троек $\{a, b, c\}$ с элементами $a, b, c \in N_{1}^{f} \backslash\{0\}$, удовлетворяющими условию

$$
a+b+c=0 \text {. }
$$


Тогда при $i=f(0)$

$$
\begin{gathered}
A f(f)=\max \left\{\frac{6}{2^{2 n}}\left(2^{n-1} k-(k-i)^{2}+4 m-\frac{i}{3}\right),\right. \\
\left.1-\frac{6}{2^{2 n}}\left(2^{n-1} k-(k-i)^{2}+4 m-\frac{i}{3}\right)\right\}, \\
A d(f)=1-\frac{6}{2^{2 n}}\left(2^{n-1} k-(k-i)^{2}+4 m-\frac{i}{3}\right) .
\end{gathered}
$$

Доказательство. Введем обозначение

$$
U_{i}=\left\{(a, b) \in V_{n}^{2}:\left|\{a, b, a+b\} \cap N_{1}^{f}\right|=i\right\}, \quad i=0,1,2,3 .
$$

Множество $V_{n}^{2}$ всех упорядоченных пар $(a, b)$ элементов из $V_{n}^{2}$ разбивается на четыре попарно не пересекающихся подмножеств $U_{i}, i=0,1,2,3$.

Очевидно, что для пар $(a, b) \in U_{0} \cup U_{2}$ и для пар $(a, b) \in U_{1} \cup U_{3}$ выполняются соответственно равенства $f(a+b)=f(a)+f(b)$ и $f(a+b)=f(a)+f(b)+1$. Следовательно,

$$
\begin{aligned}
& A d(f)=\frac{\left|U_{0} \cup U_{2}\right|}{2^{2 n}}, \\
& A f(f)=\frac{\max \left(\left|U_{0} \cup U_{2}\right|,\left|U_{1} \cup U_{3}\right|\right)}{2^{2 n}} .
\end{aligned}
$$

Так как

$$
\left|U_{0} \cup U_{1} \cup U_{2} \cup U_{3}\right|=2^{2 n}
$$

и множества $U_{0}, \ldots, U_{3}$ попарно не пересекаются, получаем, что

$$
\begin{aligned}
& A d(f)=\frac{2^{2 n}-\left|U_{1}-U_{3}\right|}{2^{2 n}}, \\
& A f(f)=\frac{\max \left(2^{2 n}-\left|U_{1} \cup U_{3}\right|,\left|U_{1} \cup U_{3}\right|\right)}{2^{2 n}} .
\end{aligned}
$$

Таким образом, для вычисления $A f(f)$ достаточно найти $\left|U_{1}\right|$ и $\left|U_{3}\right|$.

Рассмотрим сначала случай, когда $f(0)=0$, то есть $N_{1}^{f}$ не содержит нулевого вектора.

Вычислим $\left|U_{3}\right|$. Из определения $\left|U_{3}\right|$ видно, что $(a, b) \in U_{3}$ в том и только том случае, когда элементы $a, b$ содержатся в некоторой тройке из $M$. Так как $N_{1}^{f}$ не содержит нулевого вектора, компоненты каждой из таких троек попарно различны. Поэтому из каждой тройки $\{a, b, c\}$ получается 6 пар из $U_{3}:(a, b),(a, c),(b, c),(c, b),(c, a),(b, a)$. Следовательно, $\left|U_{3}\right|=6 \mathrm{~m}$.

Вычислим $\left|U_{1}\right|$. Найдем сначала число $N$ неупорядоченных троек $\{a, b, c\}$ с различными ненулевыми компонентами из $V_{n}$ и с нулевой суммой, в которых ровно одна компонента содержится в $N_{1}^{f}$. Заметим, что для каждого $c_{t} \in N_{1}^{f}$ существует ровно $2^{n-1}$ неупорядоченных пар $\{a, b\}$ таких, что $a+b=c_{t}$ (это пары вида $\left\{a, a+c_{t}\right\}$ ). Обозначим множество всех таких пар через $M_{t}$. Нас интересуют лишь те из них, в которых $a, b \notin N_{1}^{f}$. В связи с этим найдем сначала число пар $\{a, b\}$, в которых $a, b \in N_{1}^{f}$. Обозначим через $d_{t}$ число соотношений вида (12), в которые входит $c_{t}$. Каждое такое соотношение дает одну неупорядоченную пару $\left\{c_{r}, c_{s}\right\} \in M_{t}$. Всего же получим $d_{t}$ таких пар. Удалив из $M_{t}$ 
эти пары, а также пару $\left\{0, c_{t}\right\}$, получим $2^{n-1}-\left(d_{t}+1\right)$ пар, а значит, и столько же неупорядоченных троек $\left\{a, b, c_{t}\right\}$, в которых $a \neq 0, b \neq 0$ и $a \notin N_{1}^{f}$ или $b \notin N_{1}^{f}$. Суммируя числа $2^{n-1}-\left(d_{t}+1\right)$ по $t \in\{1, \ldots, k\}$, получим $\left(2^{n-1}-1\right) k-\left(d_{1}+\ldots+d_{k}\right)$. Сумма $d_{1}+\ldots+d_{k}$ равна числу вхождений всех букв из $N_{1}^{f}$ в тройки из $M$, и значит, равна $3 m$.

Таким образом, получено множество $T$ из $\left(2^{n-1}-1\right) k-3 m$ троек вида $\left\{a, b, c_{i}\right\}$, $i \in\{1, \ldots, k\}$. Из его построения видно, что оно содержит все тройки с попарно различными компонентами и с нулевой суммой компонент, в которых $c_{i}$ пробегает множество $N_{1}^{f}$, а элементы $a$ и $b$ отличны от 0 , и либо оба вектора $a, b$ не принадлежат $N_{1}^{f}$, либо только один из них принадлежит $N_{1}^{f}$, причем в последнем случае каждая тройка получена дважды. Действительно, если тройка $\left\{a, c_{i}, c_{j}\right\} \in T$, то она получена один раз при $t=i$ и один раз при $t=j$. Подсчитаем теперь число троек в $T$ с ровно двумя различными компонентами из $N_{1}^{f}$. Заметим, что при любых двух компонентах $c_{i}, c_{j} \in N_{1}^{f}$ тройки с нулевой суммой компонент ее третья компонента определяется однозначно. Однако она может принадлежать или не принадлежать $N_{1}^{f}$. Так как пара векторов $c_{i}, c_{j}$ может входить лишь в одну тройку из $M$, при всех возможных парах различных индексов $i, j$ получим $m$ различных троек с тремя компонентами из $N_{1}^{f}$, причем каждая такая тройка будет получена трижды, так как тройка $\left\{c_{r}, c_{i}, c_{j}\right\}$ будет получена при трех различных сочетаниях из чисел $r, i, j$ по 2. Отсюда следует, что число различных троек в $M$ равно $\left(\begin{array}{l}k \\ 2\end{array}\right)-3 m$, и

$$
N=\left(2^{n-1}-1\right) k-3 m-2\left(\left(\begin{array}{l}
k \\
2
\end{array}\right)-3 m\right)=\left(2^{n-1}-k\right) k+3 m .
$$

Заметим теперь, что из каждой полученной тройки $\{a, b, c\}$ получается 6 пар из множества $U_{1}$. Следовательно,

$$
\left|U_{1}\right|=6\left(\left(2^{n-1}-k\right) k+3 m\right) .
$$

Из полученных результатов находим, что

$$
\left|U_{1}\right|+\left|U_{3}\right|=6\left(\left(2^{n-1}-k\right) k+4 m\right) .
$$

Из (12) и (14) следуют утверждения теоремы в рассматриваемом случае.

Пусть теперь $f(0)=1$, то есть $N_{1}^{f}$ содержит нулевой вектор 0. Тогда все пары из $U_{3}$ без нулевых компонент строятся по тройкам из $M$, а пары, содержащие нулевые компоненты, - по тройкам $\left\{c_{i}, c_{i}, 0\right\}, i=1, \ldots, k$. При этом получится из каждой тройки без нулей 6 пар, из тройки $\left\{c_{i}, c_{i}, 0\right\} 3$ пары при $c_{i} \neq 0$ и одна пара при $c_{i}=0$. В итоге получаем, что

$$
\left|U_{3}\right|=6 m+3(k-1)+1 .
$$

Для нахождения числа $\left|U_{1}\right|$ можно поступить следующим образом. Сначала, как и в рассмотренном выше случае, найдем все тройки из $U_{1}$, содержащие по одному ненулевому вектору из $N_{1}^{f}$. Так как число таких элементов равно $k-1$, в соответствии с формулой (13) число полученных троек равно 6((2n-1 $-(k-1))(k-1)+3 m)$.

Теперь найдем все тройки из $U_{1}$, содержащие нулевые компоненты. Они исчерпываются $2^{n}-k$ тройками $\{a, a, 0\}$, где $a \neq c_{i}, i \in\{1, \ldots, k\}$. Из каждой такой тройки получается 3 пары из $U_{1}:(a, a),(a, 0),(0, a)$. В итоге получаем, что

$$
\begin{aligned}
\left|U_{1}\right| & =6\left(\left(2^{n-1}-(k-1)\right)(k-1)+3 m\right)+3\left(2^{n}-k\right), \\
\left|U_{1} \cup U_{3}\right| & =6\left(2^{n-1} k-(k-1)^{2}+4 m-1 / 3\right) .
\end{aligned}
$$


Отсюда следуют утверждения теоремы в рассматриваемом случае. В итоге теорема 2 полностью доказана.

Из условий теоремы видно, что показатель аффинности функции $f$ определяется тремя ее параметрами: числом переменных $n$, весом $k$ и числом соотношений вида (11) между ненулевыми векторами, на которых значение функции равно единице, далее это число соотношений будем обозначать через $m(f)$.

\section{4. Булевы функции с минимальным коэффициентом аффинности}

Согласно следствиям 2 и $3, A f(f) \geqslant 1 / 2$ для любой функции $f \in F_{n}$ и весьма близок к $1 / 2$ для бент-функций. С точки зрения криптографических применений представляется интересной задача описания класса булевых функции с наименьшим значением параметра $A f(f)$ в классе функций из $F_{n}$ веса $k$. В частности интересно знать, достигается ли для функций $f \in F_{n}$ оценка $1 / 2$, и существуют ли функции с меньшим коэффициентом аффинности, чем для бент-функций?

Оказывается, что оценка $1 / 2$ не достигается. Именно, справедливо следующее утверждение.

Предложение 2. Для любой функции $f \in F_{n}$ веса $k$ имеют место неравенства

$$
A f(f) \geqslant \begin{cases}\frac{1}{2}+\frac{1}{2^{2 n-3}}, & f(0)=0, k \equiv 0 \quad(\bmod 2), \\ \frac{1}{2}+\frac{1}{2^{2 n-3}}-\frac{3}{2^{2 n-1}}, & f(0)=0, k \equiv 1 \quad(\bmod 2), \\ \frac{1}{2}+\frac{1}{2^{2 n-3}}, & f(0)=1, k \equiv 0 \quad(\bmod 2), \\ \frac{1}{2}+\frac{1}{2^{2 n-3}}+\frac{3}{2^{2 n-1}}, & f(0)=1, k \equiv 1 \quad(\bmod 2),\end{cases}
$$

причем достигаться указаннье оченки могут лишь при

$$
m=m_{0}+\Delta
$$

где

$$
m_{0}=\frac{2^{2 n-4}}{3}-\frac{\left(2^{n-1}-k\right) k}{4}
$$

$u$

$$
\Delta \in\left\{-\frac{1}{3}, \frac{7}{12},-\frac{2 k-1}{4}+\frac{5}{12},-\frac{2 k-1}{4}+\frac{2}{3}\right\} .
$$

Доказательство. Из теоремы 2 следует, что для функции $f \in F_{n}, f(0)=0$, коэффициент аффинности может принять наименьшее значение $1 / 2$ лишь при таком $m(f)=m$, которое удовлетворяет равенству

$$
\frac{6}{2^{2 n}}\left(\left(2^{n-1}-k\right) k+4 m\right)=1-\frac{6}{2^{2 n}}\left(\left(2^{n-1}-k\right) k+4 m\right) .
$$


Решая это уравнение относительно $m$, получим $m=m_{0}$. Очевидно, что это число $m$ не является целым. Поэтому в данном случае оценка $1 / 2$ не достигается, и $\min \{A f(f)\}$ может получиться лишь при $m(f)=[m]$, или $m(f)=[m]+1$, где $[m]$ есть целая часть числа $m$. Легко видеть, что при четном и нечетном $k$ величина $[m]$ равна соответственно $\left(2^{2 n-4}-1\right) / 3-\left(\left(2^{n-1}-k\right) k\right) / 4$ и $\left(2^{2 n-4}\right) / 3-\left(\left(2^{n-1}-k\right) k\right) / 4-7 / 12$.

Аналогично, в случае $f(0)=1$, решая уравнение

$$
\frac{6}{2^{2 n}}\left(2^{n-1} k-(k-1)^{2}+4 m-\frac{1}{3}\right)=1-\frac{6}{2^{2 n}}\left(2^{n-1} k-(k-1)^{2}+4 m-\frac{1}{3}\right),
$$

получим, что

$$
[m]=\left[\frac{2^{2 n-4}}{3}-\frac{\left(2^{n-1}-k\right) k+2 k-1}{4}+\frac{1}{12}\right],
$$

что при четном и нечетном $k$ равно $\left(2^{2 n-4}\right) / 3-\left(\left(2^{n-1}-k\right) k+2 k-1\right) / 4-7 / 12$ и $\left(2^{2 n-4}\right) / 3-\left(\left(2^{n-1}-k\right) k+2 k-1\right) / 4-1 / 3$ соответственно.

Вычисляя теперь во всех случаях $A f(f)$ при найденных значениях $m(f)=[m]$ и $m(f)=[m]+1$, и выбирая в каждом случае минимальное значение $A f(f)$, получим соотношения из предложения 2.

Наиболее интересными для приложений являются случаи, когда $\|f\|=2^{n-1}$, то есть функция является равновероятной (сбалансированной), или $\|f\|=2^{n-1}-2^{n / 2-1}$, как у бент-функций. Из предложения 2 в этих случаях получаем следующее следствие.

Следствие 5. Пусть $f \in F_{n}, f(0)=0 u n>3$. Тогда

(а) если $\|f\|=2^{n-1}$, то для наименьшего значения величины Af( $f$ ) выполняется неравенство

$$
A f(f) \geqslant \frac{1}{2}+\frac{1}{2^{2 n-3}}
$$

и эта оченка может достигаться лишь при условии, что

$$
m=\left[\frac{2^{2 n-4}}{3}\right]=\frac{2^{2 n-4}-1}{3}
$$

(б) если $\|f\|=2^{n-1}-2^{n / 2-1}$, то для наименьшего значения величины $A f(f)$ выполняется неравенство (15), и эта оценка может достигаться лишь при

$$
m=\frac{2^{2 n-4}-1}{3}-2^{n-4}\left(2^{n / 2}-1\right) .
$$

Таким образом, для поиска булевых функций с наименьшим коэффициентом аффинности необходимо уметь строить функции с указанным в предложении 2 числом троек $m$. Вопрос о существовании таких значений параметра $m$ в общем случае остается открытым. Для $n=4,6$ примеры таких функций имеются.

Пример 1. Пусть $n=4, k=6$. Если $N_{1}^{f}=\left\{c_{1}, c_{2}, c_{3}, c_{4}, c_{5}, c_{6}\right\}$, система $\left\{c_{1}, c_{2}, c_{3}, c_{4}\right\}$ линейно независима, $c_{5}=c_{1}+c_{2}, c_{6}=c_{3}+c_{4}$, то $A f(f)=1 / 2+1 / 32=0,53125$, что совпадает с минимальным значением из части (б) следствия 5. 
Заметим, что к таким функциям относится бент-функция $x_{1} x_{2} \oplus x_{3} x_{4}$.

Вместе с тем, такой же коэффициент аффинности могут иметь и не бент-функции.

Пример 2. Пусть $n=4, k=4$. Если $N_{1}^{f}=\left\{c_{1}, c_{2}, c_{3}, c_{4}\right\}$, где система $\left\{c_{1}, c_{2}, c_{3}\right\}$ линейно независима, $c_{4}=c_{1}+c_{2}$, то $A f(f)=1 / 2+1 / 32=0,53125$.

Пример 3. Пусть $n=6, k=28, N_{1}^{g}=W_{1} \cup W_{2}$, где

$$
\begin{aligned}
& W_{1}=\{3,7,11,12,13,14,15,19,21,23,27,28,29,30\}, \\
& W_{2}=\{32,33,38,39,42,44,45,49,50,54,57,58,61,63\} .
\end{aligned}
$$

Для этой функции $\|g\|=28$ и $A f(g)=1 / 2+1 / 2^{9}$ минимален.

Пример 3 свидетельствует о том, что для $n=6$ коэффициент аффинности бентфункций не является минимальным, так как, согласно предложению 2, он для $n=6$ равен $1 / 2+1 / 2^{7}$.

\section{5. Коэффициент аффинности булевой мультилинейной функции}

В данном параграфе решается задача вычисления коэффициента аффинности для функций из класса мультилинейных функций.

Определение 3 ([2]). Функция $f \in F_{n}$ называется мультиаффинной, если она представляется в виде

$$
f(x)=\bigwedge_{i=1}^{k}\left(\left(a_{i}, x\right)+b_{i}\right),
$$

где $x, a_{i} \in V_{n}, b_{i} \in P, 1 \leqslant k \leqslant 2^{n}$.

Функции вида (16), при $b_{1}=\ldots=b_{k}=0$ будем называть мультилинейными.

Изучим, как устроен набор коэффициентов статистической структуры мультилинейной функции.

Лемма 1. Пусть

$$
f(x)=\prod_{i=1}^{k}\left(a_{i}, x\right)
$$

пусть $\left(a_{1}, \ldots, a_{r}\right), r \leqslant k,-$ максимальная линейно независимая подсистема системь векторов $\left(a_{1}, \ldots, a_{k}\right)$,

$$
a_{j}=\sum_{i=1}^{r} c_{i}^{j} a_{i}, \quad j \in\{r+1, \ldots, k\},
$$

u $c_{j}=\left(c_{1}^{j}, \ldots, c_{r}^{j}\right)$. Тогда

$$
f(x)= \begin{cases}\prod_{i=1}^{r}\left(a_{i}, x\right) & \text { если }\left\|c_{j}\right\| \equiv 1 \quad(\bmod 2) \text { для всех } j \in\{r+1, \ldots, k\}, \\ 0 & \text { если найдется } j \in\{r+1, \ldots, k\} \text { такое, что }\left\|c_{j}\right\| \equiv 0 \quad(\bmod 2) .\end{cases}
$$


Доказательство. Пусть существует вектор $c_{j}, j \in\{r+1, \ldots, k\}$, четного веса. Представим функцию $f$ в виде

$$
f(x)=\prod_{i=1}^{r}\left(a_{i}, x\right)\left(a_{j}, x\right) \prod_{\substack{i=r+1 \\ i \neq j}}^{k}\left(a_{i}, x\right) .
$$

Вектор $a_{j}$ линейно выражается через систему векторов $\left(a_{1}, \ldots, a_{r}\right)$, поэтому равенство (17) можно записать в виде

$$
f(x)=\prod_{i=1}^{r}\left(a_{i}, x\right)\left(\sum_{l=1}^{r}\left(c_{l}^{j} a_{l}, x\right)\right) \prod_{\substack{i=r+1 \\ i \neq j}}^{k}\left(a_{i}, x\right) .
$$

Поскольку вектор $c_{j}$ четного веса, верно равенство

$$
\prod_{i=1}^{r}\left(a_{i}, x\right)\left(\sum_{l=1}^{r}\left(c_{l}^{j^{\prime}} a_{l}, x\right)\right)=0
$$

и поэтому функция $f=0$.

Если вес $c_{j}$ нечетен для всех $j \in\{r+1, \ldots, k\}$, то для любого такого $j$

$$
\prod_{i=1}^{r}\left(a_{i}, x\right)\left(\sum_{l=1}^{r}\left(c_{l}^{j} a_{l}, x\right)\right)=\prod_{i=1}^{r}\left(a_{i}, x\right),
$$

и поэтому справедливо равенство

$$
f(x)=\prod_{i=1}^{r}\left(a_{i}, x\right) .
$$

Лемма доказана.

Для спектра функции $\prod_{i=1}^{r}\left(a_{i}, x\right)$ справедливо следующее утверждение.

Предложение 3. (a) Пусть

$$
f(x)=\prod_{i=1}^{r}\left(a_{i}, x\right)
$$

система векторов $\alpha=\left(a_{1}, \ldots, a_{r}\right)$ линейно независима,

$$
a=\sum_{i=1}^{r} c_{i} a_{i} \in V_{n}
$$

u $c=\left(c_{1}, \ldots, c_{r}\right)$. Тогда

$$
\Delta_{a}^{f}=\left\{\begin{array}{lll}
2^{n-1}-2^{n-r}, & a=0 \\
2^{n-r}, & \|c\| \equiv 0 & (\bmod 2), a \neq 0 \\
-2^{n-r}, & \|c\| \equiv 1 & (\bmod 2), a \neq 0
\end{array}\right.
$$


(б) Если $a \in V_{n}$ и система $\left(a_{1}, \ldots, a_{r}, a\right)$ линейно независима, то $\Delta_{a}^{f}=0$.

При этом числа равных элементов спектра находятся по формулам

$$
\begin{aligned}
\left|\left\{a \in V_{n}: \Delta_{a}^{f}=2^{n-1}-2^{n-r}\right\}\right| & =1 ; \\
\left|\left\{a \in V_{n}: \Delta_{a}^{f}=2^{n-r}\right\}\right| & =2^{r-1} ; \\
\left|\left\{a \in V_{n}: \Delta_{a}^{f}=-2^{n-r}\right\}\right| & =2^{r-1}-1 ; \\
\mid\left\{a \in V_{n}: \Delta_{a}^{f}=0\right\} \| & =2^{n}-2^{r} .
\end{aligned}
$$

Доказательство. Воспользуемся определением коэффициента статистической структуры в форме (1).

Так как система $\alpha$ линейно независима, верно равенство $\|f\|=2^{n-r}$, и в случае $a=0$ утверждение верно. Вычислим вес функции $f(x)(a, x)$ при $a \neq 0$.

Из доказательства леммы 1 следует система равенств

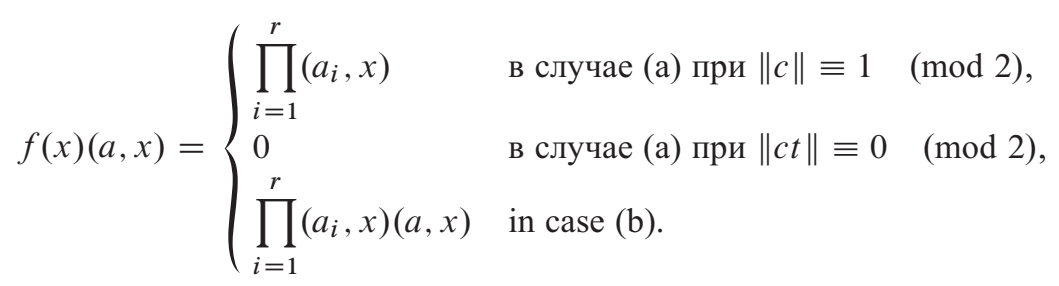

Отсюда следует, что

$$
\|f(x)(a, x)\|=\left\{\begin{array}{lll}
2^{n-r} & \text { в случае (а) при }\|c\| \equiv 1 & (\bmod 2), \\
0 & \text { в случае (а) при }\|c\| \equiv 0 & (\bmod 2), \\
2^{n-(r+1)} & \text { в случае (б). }
\end{array}\right.
$$

Подставляя полученные значения в формулу (1), получим искомые равенства для коэффициентов статистической структуры функции $f(x)$. Из них нетрудно вычислить и кратности каждого из элементов спектра функции $f$. В итоге утверждение доказано.

Пользуясь значениями коэффициентов статистической структуры функции $f$, вычислим величину коэффициента аффинности произвольной мультилинейной функции.

Предложение 4. Пусть

$$
f(x)=\prod_{i=1}^{k}\left(a_{i}, x\right),
$$

$u\left(a_{1}, \ldots, a_{r}\right), r \leqslant k \leqslant 2^{n}$, есть максимальная линейно независимая подсистема системь векторов $\left(a_{1}, \ldots, a_{k}\right)$. Тогда в обозначениях леммь 1

$$
A f(f)=\max \left\{\frac{1}{2} \pm \frac{2^{2 r-2}-3\left(2^{r-1}-1\right)}{2^{2 r-1}}\right\}
$$

если $\left\|c_{j}\right\| \equiv 1(\bmod 2)$ для всех $j \in\{r+1, \ldots, k\}, u A f(f)=1$, если найдется $j \in$ $\{r+1, \ldots, k\}$ такое, что $\left\|c_{j}\right\| \equiv 0(\bmod 2)$.

Справедливость предложения 4 следует из леммы 1, предложения 3 и следствия 3. 


\section{6. Коэффициент аффинности функций, близких к бент-функциям}

Рассмотрим задачу нахождения коэффициента аффинности функции, отличающейся от бент-функции на фиксированном наборе векторов. Для двух различных функций $f, g \in F_{n}$ введем обозначения

$$
\begin{aligned}
& V_{n}^{(0)}(f, g)=\left\{x \in V_{n}: f(x)=g(x)\right\}, \\
& V_{n}^{(1)}(f, g)=V_{n} \backslash V_{n}^{(0)}(f, g),
\end{aligned}
$$

и установим связь между коэффициентами $A d(f)$ и $A d(g)$.

Для этого нам потребуется понятие автокорреляционной функции для булевой функции (см., например, [3]).

Определение 4. Автокорреляционной функцией функции $f \in F_{n}$ называется функция $\Delta_{f}: V_{n} \rightarrow \mathbf{Z}$, определенная формулой

$$
\Delta_{f}(u)=\sum_{x \in V_{n}}(-1)^{f(x+u)+f(x)} .
$$

Предложение 5. Для любых различных функций $f, g \in F_{n}$

$$
\begin{aligned}
& A d(f)-A d(g) \\
& =\frac{3}{2^{2 n}} \sum_{z \in V_{n}^{(1)}(f, g)}(-1)^{f(z)} \Delta_{f}(z) \\
& \quad-\frac{3}{2^{2 n-1}} \sum_{z \in V_{n}^{(1)}(f, g)} \sum_{y \in V_{n}^{(1)}(f, g)}(-1)^{f(z)+f(y)+f(z+y)} \\
& \quad+\frac{1}{2^{3 n-2}} \sum_{x \in V_{n}^{(1)}(f, g)} \sum_{y \in V_{n}^{(1)}(f, g)} \sum_{z \in V_{n}^{(1)}(f, g)}(-1)^{f(z)+f(y)+f(x)} \sum_{\alpha \in V_{n}}(-1)^{(\alpha, z+y+x) .}
\end{aligned}
$$

Доказательство. В соответствии с формулой (17),

$$
A d(f)-A d(g)=\frac{1}{2^{3 n-2}} \sum_{a \in V_{n}}\left(\left(\Delta_{a}^{f}\right)^{3}-\left(\Delta_{a}^{g}\right)^{3}\right)
$$

Вычислим величину одного слагаемого при любом фиксированном значении вектора $a$. По формуле разности кубов

$$
\left(\Delta_{a}^{f}\right)^{3}-\left(\Delta_{a}^{g}\right)^{3}=\left(\Delta_{a}^{f}-\Delta_{a}^{g}\right)\left(\left(\Delta_{a}^{f}\right)^{2}+\Delta_{a}^{f} \Delta_{a}^{g}+\left(\Delta_{a}^{g}\right)^{2}\right) .
$$

По определению 4 , учитывая, что $\Delta_{a}^{f}-\Delta_{a}^{g}=0$ при $a \in V_{n}^{(0)}(f, g)$, получим, что

$$
\begin{aligned}
\Delta_{a}^{f}-\Delta_{a}^{g} & =\frac{1}{2}\left(\sum_{x \in V_{n}^{(1)}(f, g)}\left((-1)^{f(x)+(a, x)}-(-1)^{g(x)+(a, x)}\right)\right) \\
& =\sum_{x \in V_{n}^{(1)(f, g)}}(-1)^{f(x)+(a, x)} .
\end{aligned}
$$


Проводя аналогичные рассуждения, вычислим второй сомножитель:

$$
\begin{aligned}
\left(\Delta_{a}^{f}\right)^{2}+\Delta_{a}^{f} \Delta_{a}^{g}+\left(\Delta_{a}^{g}\right)^{2}=\frac{3}{4} & \sum_{x \in V_{n}^{(0)}(f, g)} \sum_{y \in V_{n}^{(0)}(f, g)}(-1)^{f(x)+f(y)+(a, x+y)} \\
& +\frac{1}{4} \sum_{x \in V_{n}^{(1)}(f, g)} \sum_{y \in V_{n}^{(1)}(f, g)}(-1)^{f(x)+f(y)+(a, x+y) .}
\end{aligned}
$$

Полученную формулу можно упростить. Обозначим

$$
c_{x, y}=(-1)^{f(x)+f(y)+(a, x+y)} .
$$

Прибавим к сумме $\sum_{x \in V_{n}^{(0)}(f, g)} \sum_{y \in V_{n}^{(0)}(f, g)} c_{x, y}$ и вычтем из нее одну и ту же величину $\sum_{x \in V_{n}^{(0)}(f, g)} \sum_{y \in V_{n}^{(1)}(f, g)} c_{x, y}+\sum_{x \in V_{n}^{(1)}(f, g)} \sum_{y \in V_{n}^{(0)}(f, g)} c_{x, y}+\sum_{x \in V_{n}^{(1)}(f, g)} \sum_{y \in V_{n}^{(1)}(f, g)} c_{x, y}$. Тогда, в соответствии с определением коэффициента статистической структуры булевой функции, получим, что

$$
\begin{aligned}
\sum_{x \in V_{n}^{(0)}(f, g)} \sum_{y \in V_{n}^{(0)}(f, g)} c_{x, y}=4\left(\Delta_{\alpha}^{f}\right)^{2}-2 \sum_{x \in V_{n}^{(0)}(f, g)} \sum_{y \in V_{n}^{(1)}(f, g)} c_{x, y} & -\sum_{x \in V_{n}^{(1)}(f, g)} \sum_{y \in V_{n}^{(1)}(f, g)} c_{x, y} .
\end{aligned}
$$

Произведя соответствующую замену в формуле (18), получим равенство

$$
\begin{array}{r}
\left(\Delta_{a}^{f}\right)^{2}+\Delta_{a}^{f} \Delta_{a}^{g}+\left(\Delta_{a}^{g}\right)^{2}=\frac{1}{4}\left(12\left(\Delta_{a}^{f}\right)^{2}-6 \sum_{x \in V_{n}^{(0)}(f, g)} \sum_{y \in V_{n}^{(1)}(f, g)} c_{x, y}\right. \\
\left.\quad-2 \sum_{x \in V_{n}^{(1)}(f, g)} \sum_{y \in V_{n}^{(1)}(f, g)} c_{x, y}\right) .
\end{array}
$$

Преобразуем правую часть (19) к виду $3\left(\Delta_{a}^{f}\right)^{2}-3 \Delta_{a}^{f} \sum_{y \in V_{n}^{(1)}(f, g)}(-1)^{f(y)+(a, y)}+$ $\sum_{x \in V_{n}^{(1)}(f, g)} \sum_{y \in V_{n}^{(1)}(f, g)}(-1)^{f(x)+f(y)+(\alpha, x+y)}$ и получим

$$
\begin{aligned}
\left(\Delta_{a}^{f}\right)^{3}-\left(\Delta_{a}^{g}\right)^{3}= & \sum_{z \in V_{n}^{(1)}(f, g)}(-1)^{f(z)+(a, z)}\left(3\left(\Delta_{a}^{f}\right)^{2}-3 \Delta_{a}^{f} \sum_{y \in V_{n}^{(1)}(f, g)}(-1)^{f(y)+(a, y)}\right. \\
& \left.+\sum_{x \in V_{n}^{(1)}(f, g)} \sum_{y \in V_{n}^{(1)}(f, g)}(-1)^{f(x)+f(y)+(a, x+y)}\right) .
\end{aligned}
$$

Просуммируем полученное выражение по всем значениям вектора $a$. Для этого вычислим предварительно суммы

$$
\begin{aligned}
& S_{1}=\sum_{a \in V_{n}} \sum_{z \in V_{n}^{(1)}(f, g)}(-1)^{f(z)+(a, z)}\left(\Delta_{a}^{f}\right)^{2}, \\
& S_{2}=\sum_{a \in V_{n}} \sum_{z \in V_{n}^{(1)}(f, g t)} \sum_{y \in V_{n}^{(1)}}(f, g)(-1)^{f(z)+f(y)+(a, z+y)} \Delta_{a}^{f} .
\end{aligned}
$$


Используя формулу разложения функции $f$ по характерам группы $V_{n}$ над полем комплексных чисел, получим, что

$$
\begin{aligned}
& S_{1}=\frac{1}{4} \sum_{x \in V_{n}} \sum_{y \in V_{n}} \sum_{z \in V_{n}^{(1)}(f, g)}(-1)^{f(x)+f(y)+f(z)} \sum_{a \in V_{n}}(-1)^{(a, x+y+z)}, \\
& S_{2}=\frac{1}{2} \sum_{x \in V_{n}} \sum_{y \in V_{n}^{(1)}(f, g)} \sum_{z \in V_{n}^{(1)}(f, g)}(-1)^{f(x)+f(y)+f(z)} \sum_{a \in V_{n}}(-1)^{(a, x+y+z)} .
\end{aligned}
$$

Сумма $\sum_{a \in V_{n}}(-1)^{(a, x+y+z t)}$ равна $2^{n}$ в случае $x+y+z=0$ и 0 в остальных случаях. Учитывая данное свойство, приходим к формулам

$$
\begin{aligned}
& S_{1}=\frac{2^{n}}{4} \sum_{z \in V_{n}^{(1)}(f, g)}(-1)^{f(z)} \Delta_{f}(z), \\
& S_{2}=2^{n-1} \sum_{z \in V_{n}^{(1)}(f, g)} \sum_{y \in V_{n}^{(1)}(f, g)}(-1)^{f(z)+f(y)+f(z+y) .}
\end{aligned}
$$

Умножив найденные выражения на соответствующие коэффициенты, получим доказываемое предложение.

Рассмотрим некоторые частные случаи.

Следствие 6. Если множество $V_{n}^{(1)}(f, g)$ является подпространством в $V_{n}$, то

$$
\begin{aligned}
A d(f)-A d(g)=\frac{3}{2^{2 n}} \sum_{z \in V_{n}^{(1)}(f, g)}(-1)^{f(z)} \Delta_{f}(z) & \\
& -\frac{1}{2^{2 n-1}} \sum_{z \in V_{n}^{(1)}(f, g)} \sum_{y \in V_{n}^{(1)}(f, g)}(-1)^{f(z)+f(y)+f(z+y) .}
\end{aligned}
$$

Пусть далее $f-$ бент-функция. Тогда

$$
\frac{3}{2^{2 n}} \sum_{z \in V_{n}^{(1)}(f, g)}(-1)^{f(z)} \Delta_{f}(z)= \begin{cases}\frac{3}{2^{n}(-1)^{f(0)}} & 0 \in V_{n}^{(1)}(f, g) \\ 0 & 0 \notin V_{n}^{(1)}(f, g)\end{cases}
$$

Для любого значения $i \in\left\{1, \ldots, 2^{n-1}\right\}$ зафиксируем $2^{n}-i$ векторов. Функцию, совпадающую с бент-функцией $f$ на данном наборе векторов обозначим через $f_{i}$. Отметим, что дальнейшие рассуждения не зависят от набора фиксируемых векторов.

Для значений параметра $i=1,2,3$ вычислим значение разности коэффициентов аддитивности. 
Следствие 7. Если $f-$ бент-функичия, то

$$
\begin{aligned}
& A d(f)-A d\left(f_{1}\right)= \begin{cases}\frac{3 \cdot 2^{n-1}-1}{2^{2 n-1}}(-1)^{f(0)}, & V_{n}^{(1)}\left(f, f_{1}\right)=\{0\}, \\
-\frac{3}{2^{2 n-1}}(-1)^{f(0)}, & V_{n}^{(1)}\left(f, f_{1}\right)=\{\gamma\} ;\end{cases} \\
& A d(f)-A d\left(f_{2}\right)= \begin{cases}\frac{3 \cdot 2^{n-1}-4}{2^{2 n-1}}(-1)^{f(0)}, & V_{n}^{(1)}\left(f, f_{2}\right)=\{0, \gamma\}, \\
-\frac{6}{2^{2 n-1}}\left((-1)^{f(0)}\right. & \\
\left.+(-1)^{f\left(\gamma_{1}\right)+f\left(\gamma_{2}\right)+f\left(\gamma_{1}+\gamma_{2}\right)}\right), & V_{n}^{(1)}\left(f, f_{2}\right)=\left\{\gamma_{1}, \gamma_{2}\right\} ;\end{cases}
\end{aligned}
$$

$u$

$$
\begin{aligned}
& \operatorname{Ad}(f)-A d\left(f_{3}\right)= \\
& \qquad \begin{cases}\frac{3 \cdot 2^{n-1}-7}{2^{2 n-1}}(-1)^{f(0)} & V_{n}^{(1)}\left(f, f_{3}\right)=\left\{0, \gamma_{1}, \gamma_{2}\right\}, \\
-\frac{6}{2^{2 n-1}}(-1)^{f\left(\gamma_{1}\right)+f\left(\gamma_{2}\right)+f\left(\gamma_{1}+\gamma_{2}\right)}, & \\
-\frac{3}{2^{2 n-1}}\left((-1)^{f(0)}\right. & V_{n}^{(1)}\left(f, f_{3}\right)=\left\{\gamma_{1}, \gamma_{2}, \gamma_{3}\right\}, \\
\left.+2(-1)^{f\left(\gamma_{1}\right)+f\left(\gamma_{2}\right)+f\left(\gamma_{3}\right)}\right), & \gamma_{1}+\gamma_{2}=\gamma_{3}, \\
-\frac{3}{2^{2 n-1}}\left(3(-1)^{f(0)}\right. & \\
\left.+2 \sum_{1 \leqslant i<j \leqslant 3}(-1)^{f\left(\gamma_{3}\right)+f\left(\gamma_{j}\right)+f\left(\gamma_{3}+\gamma_{j}\right)}\right), & V_{n}^{(1)}\left(f, f_{3}\right)=\left\{\gamma_{1}, \gamma_{2}, \gamma_{3}\right\}\end{cases}
\end{aligned}
$$

\section{Список литературы}

1. Амбросимов А. С., Свойства бент-функций $q$-значной логики над конечными полями. Дискретная математика (1994) 6, №3, 50-60.

2. Горшков С. П., Применение теории $N P$-полных задач для оценки сложности решения систем булевых уравнений. Обозрение прикладной и промышленной математики (1995) 2, 325-399.

3. Логачев О. А., Сальников А. А., Ященко В. В., Булевы функиии в теории кодирования и криптологии. МЦНМО, Москва, 2004.

4. Солодовников В. И., Бент-функции из конечной абелевой группы в конечную абелеву группу. Дискретная математика (2002) 14, №1, 99-113.

5. Bellare M., Goldwasser S., Lund C., Russell A., Efficient probabilistic checkable proofs and applications to approximation. In: Proc. STOC'94. ACM, New York, 1994, p. 820.

6. Bellare M., Coppersmith D., Hastad J., Kiwi M., Sudan M., Linearity testing in characteristic two. IEEE Trans. Inform. Theory (1996) 42, 1781-1795.

7. Blum M., Luby M., Rubinfeld R., Self-testings/correcting with applications to numerical problems. J. Comput. Syst. Sci. (1993) 47, 549-595.

8. Golomb S. W., On classification of Boolean functions. IRE Trans. Circuit Theory (1959) 6, 176-186.

9. Kaufman T., Litsyn S., Xie N., Breaking the $\epsilon$-soundness bound of the linearity test over $G F(2)$. SIAM J. Comput. (2010) 39, 1998-2003. 